\title{
Características Anatómicas y Biométricas del Apéndice Vermiforme en Niños Chilenos Operados por Apendicitis Aguda
}

\author{
Anatomic and Biometric Features of the Vermiform Appendix \\ in Chilean Children Operated by Acute Appendicitis
}

\author{
*Rodrigo Verdugo \& ${ }^{* *}$ Enrique Olave
}

\begin{abstract}
VERDUGO, R. \& OLAVE, E. Características anatómicas y biométricas del apéndice vermiforme en niños chilenos operados por apendicitis aguda. Int. J. Morphol., 28(2):615-622, 2010.

RESUMEN: Las características biométricas del apéndice vermiforme no son constantes en el ser humano, así como tampoco lo es su posición. Los datos sobre sus variaciones aún son limitados, pero muestran la existencia de diferencias dependiendo de la región geográfica y racial de los individuos. En Latinoamérica no existen estudios anatómicos in vivo publicados por lo que las referencias se basan sólo en observaciones post mortem. El presente estudio tuvo como objetivo determinar y describir las características biométricas y posiciones anatómicas del apéndice vermiforme. Para ello se realizaron observaciones directas y mediciones del apéndice vermiforme en 65 niños chilenos, 37 de sexo masculino y 28 del femenino, operados por apendicitis aguda en los hospitales regionales del Maule y Aysén. Se consideraron variables como: ubicación del ciego, posición y longitud del apéndice, forma y número de ramas arteriales del mesoapéndice. La edad y peso promedio fue de 9,7 años y $37,5 \mathrm{~kg}$., respectivamente. El ciego se localizó en la fosa ilíaca derecha en 62 pacientes $(95,4 \%)$ y en el flanco derecho en $3(4,6 \%)$. La longitud promedio del apéndice fue de 7,5 cm. La posición más frecuente del apéndice vermiforme fue la retrocecal (47\%), seguida de la pélvica (29\%). El mesoapéndice fue mayoritariamente triangular, con tres ramas arteriales y se insertó principalmente en el tercio medio del apéndice. La vía de abordaje más frecuente fue la laparotomía de McBurney, seguida de la laparoscópica. La vía de abordaje laparoscópica presenta ventajas relacionadas con un menor tamaño de incisión cutánea, mejor exploración de la cavidad peritoneal, visualización más cercana de los órganos, mayor facilidad en la disección y extracción del apéndice con recuperación más rápida y estadía hospitalaria más corta.
\end{abstract}

PALABRAS CLAVE: Apéndice vermiforme; Anatomía; Biometría.

\section{INTRODUCCIÓN}

La población pediátrica chilena, es decir los menores de 15 años, al igual que la de otros países sufre de distintas enfermedades. Dentro de ellas se encuentran las que requieren de resolución quirúrgica, ya sea en forma electiva o de urgencia. Entre estas últimas, una de las patologías más prevalentes la constituye la Apendicitis Aguda (Rostion, 2001), la cual corresponde a proceso agudo primeramente inflamatorio y luego infeccioso del apéndice vermiforme (Ashcraft et al., 2002).

Tanto del punto de vista epidemiológico como de salud pública, la Apendicitis Aguda tiene una enorme repercusión en la población infantil. No sólo tiene un alto riesgo para la salud de quien la padece, debido a que sus complicaciones pueden llevar a causar la muerte, sino que también provoca un prolongado ausentismo escolar, ocupa gran volumen de recursos médicos hospitalarios y genera alto estrés familiar, entre muchas otras consecuencias (Ashcraft \& Holder, 1995).

El principal proceso patológico que afecta al apéndice vermiforme es la Apendicitis Aguda, que consiste en la inflamación con edema y exudación de las capas internas del órgano, lo que ocurre principalmente como consecuencia de la oclusión de su lumen secundariamente a hiperplasia linfoide, fecalitos, parásitos, cuerpos extraños y tumores. El tratamiento quirúrgico de la apendicitis aguda se basa en realizar una apendicectomía (extirpación del apéndice vermiforme), lo que permite el control del foco causante primario. Un punto de controversia entre los especialistas ha

\footnotetext{
* Hospital Regional de Coyhaique; Programa de Magister em Ciencias, mención Morfología, Universidad de La Frontera, Temuco, Chile.

${ }^{*}$ Facultad de Medicina, Universidad de La Frontera, Temuco, Chile.
} 
sido la ubicación anatómica del apéndice vermiforme y la posición y dimensión de la laparotomía (Cárdenas, 1992; Sabiston, 1996; Moore \& Dalley, 2002). Además del clásico punto y abordaje quirúrgico de McBurney en la fosa ilíaca derecha (McBurney,1894), existen otras incisiones como la Transversa, Paramediana y la Media infraumbilical (Sabiston).

Hoy en día se proponen en forma cada vez más frecuente, incisiones más pequeñas y más bajas, cercanas a la cadera y región inguinal, así como también la extirpación a través del ombligo (Pestana-Tirado \& Moreno, 2004).

Por otra parte, el conocimiento de la región abdominal y en particular del apéndice vermiforme por parte de los cirujanos ha sido adquirido fundamentalmente por el estudio de textos clásicos de anatomía quirúrgica y por su propia experiencia.

Con el desarrollo de la cirugía laparoscópica -mediante la cual se obtiene una visión de mejor calidad- se comenzó a explorar in vivo de mejor forma el abdomen, identificando claramente las estructuras y órganos (Nyhus et al.,1999). Ello ha permitido confirmar lo que muchos anatomistas y cirujanos señalan, en el sentido de que en ocasiones el Apéndice vermiforme puede ubicarse en diferentes posiciones relacionadas al ciego (Sabiston; Netter, 1999; Moore \& Dalley; Ndoye et al., 2005) y que éste, a su vez, no siempre se encuentra en la fosa ilíaca derecha (Netter). Lo anterior apoyaría la idea de que no siempre se debe abordar la cirugía con la misma incisión.

En relación con su morfología, el apéndice vermiforme corresponde a un divertículo intestinal que nace del ciego, situado intraperitonealmente en el cuadrante más bajo y derecho del abdomen, conocido como fosa ilíaca derecha (Sabiston; Williams et al.,1995). Habitualmente, el punto de nacimiento en el ciego es más o menos constante y está situado generalmente en el punto de conjunción de las tenias que se encuentran en la superficie del intestino grueso (Sabiston; O'Neill et al., 1998). Sin embargo, la punta del apéndice puede estar en distintas posiciones. Las más descritas son retrocecal, subcecal, pélvica, preileal y retroileal.

El apéndice vermiforme está conectado por un mesoapéndice corto con la parte más posterior del mesenterio del ileón terminal. Este meso es generalmente triangular, extendiéndose a lo largo del apéndice hasta casi su extremo distal. El mesoapéndice tiene un margen libre que lleva los vasos sanguíneos, representados por la arteria apendicular, rama de la arteria ileocólica y drena su sangre a través de la vena ileocólica, tributaria de la mesentérica superior (Moore \& Dalley).
La epidemiología de la apendicitis aguda en Chile no difiere del resto del mundo, siendo la principal causa de cirugía de urgencia en la población pediátrica (Rostion). Sin embargo, no existen estudios clínicos publicados que pongan el énfasis desde el punto de vista anatómico y que entreguen información sobre la morfología y posición del apéndice vermiforme en población chilena

Basado en los diferentes resultados obtenidos por los autores consultados, el presente estudio pretende describir las características anatómicas y biométricas del apéndice vermiforme en niños chilenos operados por apendicitis aguda.

\section{MATERIAL Y MÉTODO}

El estudio se diseñó de manera descriptiva y prospectiva. Las muestras utilizadas como material para el estudio corresponden a apéndices vermiformes observados in vivo en niños chilenos de ambos sexos, operados por apendicitis aguda en los Hospitales Regionales de Talca y Coyhaique entre Enero de 2006 y Junio de 2008. Corresponden a treinta y siete varones (37) y a veintiocho mujeres (28), cuyas edades fluctuaron entre tres y dieciséis años. Los promedios de peso y talla fueron $37,5 \pm 15,1 \mathrm{~kg}$ y 136,3 $\pm 20,9 \mathrm{~cm}$, respectivamente.

Los pacientes incluidos en el estudio correspondieron a los niños que consultaron y fueron operados por sospecha de tener apendicitis aguda, en el servicio de urgencia de los hospitales regionales de Talca y Coyhaique, durante turnos que realizó el cirujano asignado para el estudio, el cual estaba en conocimiento de los objetivos de la investigación y conocía las variables a evaluar. En el caso del Hospital Regional de Talca en el período comprendido entre Octubre de 2005 a Diciembre de 2006 fueron incluidos 38 pacientes. En el caso del Hospital Regional de Coyhaique en el período comprendido entre Febrero de 2007 y Junio de 2008 fueron incluidos 27 pacientes.

Dado que los pacientes eran intervenidos por una patología aguda se obtuvo autorización para realizar la cirugía mediante la firma de consentimiento de parte de sus padres o tutores. En forma adicional, para incluir a los pacientes específicamente en el estudio se solicitó paralelamente una autorización mediante la firma de un segundo consentimiento, el cual fue diseñado exclusivamente para el estudio y aprobado previamente por el comité de ética científica del Servicio de Salud.

Los pacientes fueron preparados para realizar ci- 
rugía según protocolo en caso de sospecha de apendicitis aguda.

Una vez hecha las determinaciones descritas, se procedió realizar la apendicectomía. La vía de abordaje quirúrgico determinada en este estudio fue principalmente mediante laparotomía de McBurney en el $66 \%$ de los casos, seguida de técnica Laparoscópica en el 24\%. Una vez en la cavidad abdominal, se disecó primeramente el mesoapéndice hasta la base del apéndice y se ligó este último con ligadura de sutura reabsorbible a aproximadamente medio centímetro de su punto de unión con el ciego. Una vez seccionado, se extrajo el apéndice vermiforme y en forma extracorpórea se procedió a realizar su medición con regla ad hoc. Se continuó la cirugía realizando lavado con suero fisiológico del área afectada, se revisó la hemostasia y finalmente se procedió al cierre de la laparotomía mediante el uso de suturas quirúrgicas específicas.

Tanto en el intraoperatorio como en el manejo del apéndice extirpado se tomaron fotografías con cámara digital Sony, modelo cibershot.

Una vez finalizada la medición del apéndice vermiforme, éste fue almacenado en un frasco con formaldehído al $10 \%$ y enviado a estudio de anatomía patológica (procedimiento de rutina con este tipo de piezas operatorias). En una pauta se registraron las siguientes variables:

I. Paciente ( edad, talla y peso).

II. Apéndice cecal.

1. Ubicación del ciego (fosa ilíaca derecha, flanco derecho u otro).

2. Origen (post valva ileocecal, ángulo inferior del ciego, cara posterolateral del ciego, extremo superior del ciego, otro).

3. Longitud.

4. Ubicación del extremo distal (retrocecal, subcecal, pélvico, retroileal, preileal).

III. Arteria apendicular.

1. Número de ramas.

IV. Mesoapéndice.

1. Forma (triangular u ovalado).

2. Inserción en el apéndice (tercio medio, tercio distal).

\section{RESULTADOS}

En relación a los 65 pacientes estudiados, 37 de ellos correspondieron a varones, lo que representa el 56,9\% de la muestra y 28 a mujeres, que representa el 43,1\%.

En relación a la distribución por sexo en ambas regiones, en la Región del Maule los varones fueron 25 (67,5 $\%)$ y las mujeres fueron $12(32,5 \%)$. En tanto en la Región de Aysén los varones fueron 12 ( 42,9\%) y las mujeres fueron $16(57,1 \%)$.

El ciego se localizó en la fosa ilíaca derecha en 62 pacientes $(95,4 \%)$. De ellos $36(55,4 \%)$ fueron de sexo masculino y 26 (40\%) de sexo femenino; en el flanco derecho se observó en 3 pacientes $(4,6 \%)$, de los cuales 1 fue de sexo masculino $(1,5 \%)$ y 2 fueron de sexo femenino $(3,1 \%)$.

El punto de origen del apéndice vermicular se encontró a nivel del punto más inferior del ciego en 21 casos $(32,3 \%)$; posterior a la valva ileocecal en $43(66,2 \%)$ y posterolateral en un caso $(1,5 \%)$. No se encontraron casos de origen en la parte superior del ciego.

La distribución del punto de origen del apéndice vermiforme por sexo fue la siguiente: en el sexo masculino el punto de origen del apéndice se observó a nivel del punto más inferior del ciego en 10 casos $(27 \%)$ y posterior a la valva ileocecal en 27 (73\%); en el sexo femenino, se observó a nivel del ángulo inferior del ciego en 11 casos (39,3 $\%)$; posterior a la valva ileocecal en $16(57,1 \%)$ y a nivel posterolateral del ciego en $1(3,6 \%)$. La Fig. 1 muestra el origen del apéndice vermiforme en el ciego.

La longitud del apéndice fue en promedio 7,56 \pm 1,63 $\mathrm{cm}$. En la Fig. 2 se muestra una pieza operatoria extirpada para registrar su longitud. En el sexo femenino fue de 7,6 \pm $1,81 \mathrm{~cm}$ y en el masculino de 7,54 $\pm 1,52 \mathrm{~cm}$. Al comparar esta longitud entre sexos, no hubo diferencia estadísticamente significativa. En la Fig. 3 se muestra la distribución de la longitud del apéndice, por sexos.

Con respecto a la posición de la punta del apéndice vermiforme, éste se ubicó en forma retrocecal en 31 pacientes (47,7\%); pélvico en 19 (29,2\%); preileal en 11 $(16,9 \%)$ y subcecal en $4(6,2 \%)$. En la Fig. 4 se muestran las distintas posiciones que puede adoptar el apéndice vermiforme.

Al determinar esta característica por sexo, el resultado mantuvo el mismo orden de frecuencia siendo el siguiente: en el femenino fue retrocecal en 11 casos $(39,3 \%)$, respecto al total de pacientes con este sexo; pélvico en 10 (35,7\%); preileal en $5(17,9 \%)$ y subcecal en $2(7,1 \%)$; en el sexo masculino fue retrocecal en 20 casos $(54,1 \%)$; pélvico en 9 $(24,3 \%)$; preileal en $6(16,2 \%)$ y subcecal en $2(5,4 \%)$.

Respecto a la posición del apéndice, considerando el origen geográfico de los pacientes, en los provenientes de la Región del Maule (38) se observó que fue retrocecal en 21 casos $(55,3 \%)$, pélvico en $7(18,4 \%)$, preileal en $6(15,8 \%)$ y subcecal en 4 (10,5\%); en los provenientes de la Región 


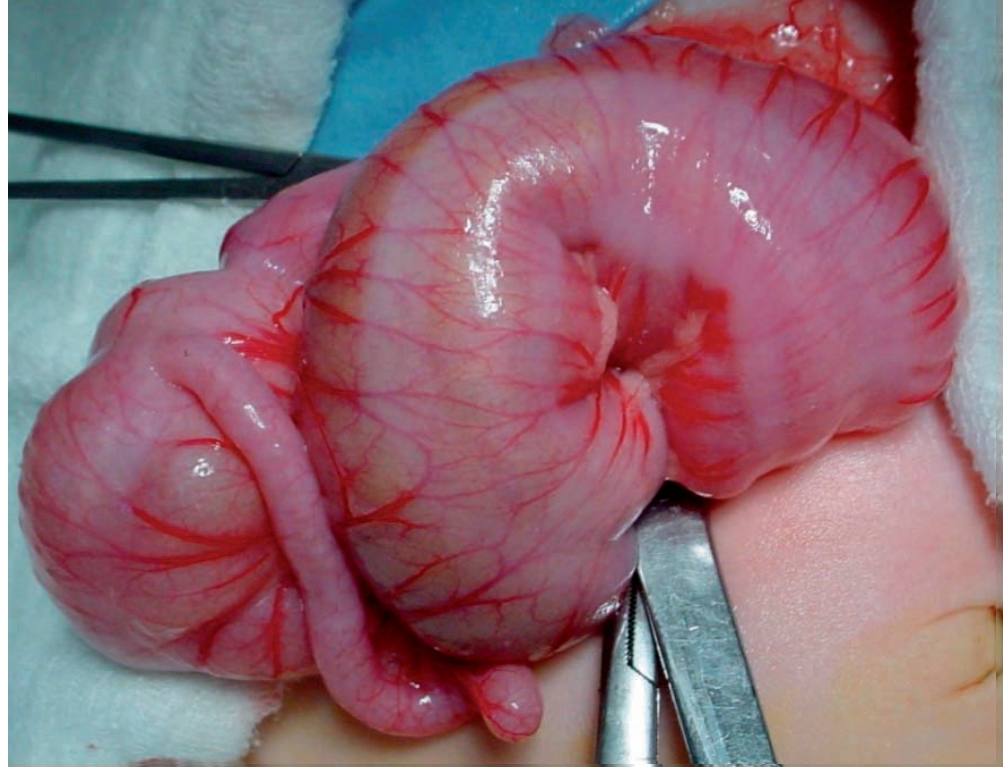

Fig. 1. Origen del apéndice vermiforme en el ciego.

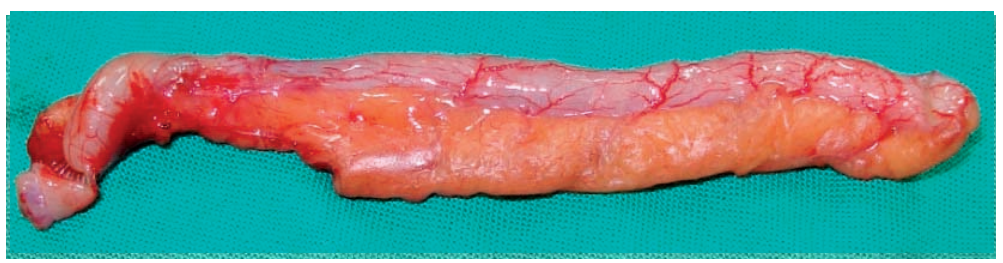

Fig. 2. Apéndice vermiforme en posición para registrar su longitud. de Aysén (27) se encontró que fue retrocecal en 10 casos $(37 \%)$, pélvico en $12(44,4 \%)$ y preileal en $5(18,5 \%)$.

Al considerar a los pacientes por sexo, los provenientes de la Región del Maule tuvieron la siguiente distribución: en el masculino (25) se encontró al apéndice en posición retrocecal en 14 casos $(56 \%)$, pélvica en $5(20 \%)$, preileal en $4(16 \%)$ y subcecal en 2 (8\%); en el femenino (13) se observó retrocecal en 7 casos $(53,8 \%)$, pélvica en 2 $(15,4 \%)$, preileal en $2(15,4 \%)$ y subcecal en $2(15,4 \%)$.

Los pacientes provenientes de la Región de Aysén presentaron la siguiente distribución: en el masculino (12) se observó en posición retrocecal en 6 casos $(50 \%)$, pélvica en $4(33,3 \%)$ y preileal en $2(16,75)$; en el femenino (15) fue retrocecal en 4 casos $(26,7 \%)$, pélvico en $8(53,3 \%)$ y preileal en $3(20 \%)$.

Respecto de la variable estatura de los niños incluidos en la muestra, al correlacionarla con la variable longitud del apéndice de los pacientes estudiados, utilizando el test de Pearson, no hubo correlación entre estas variables.

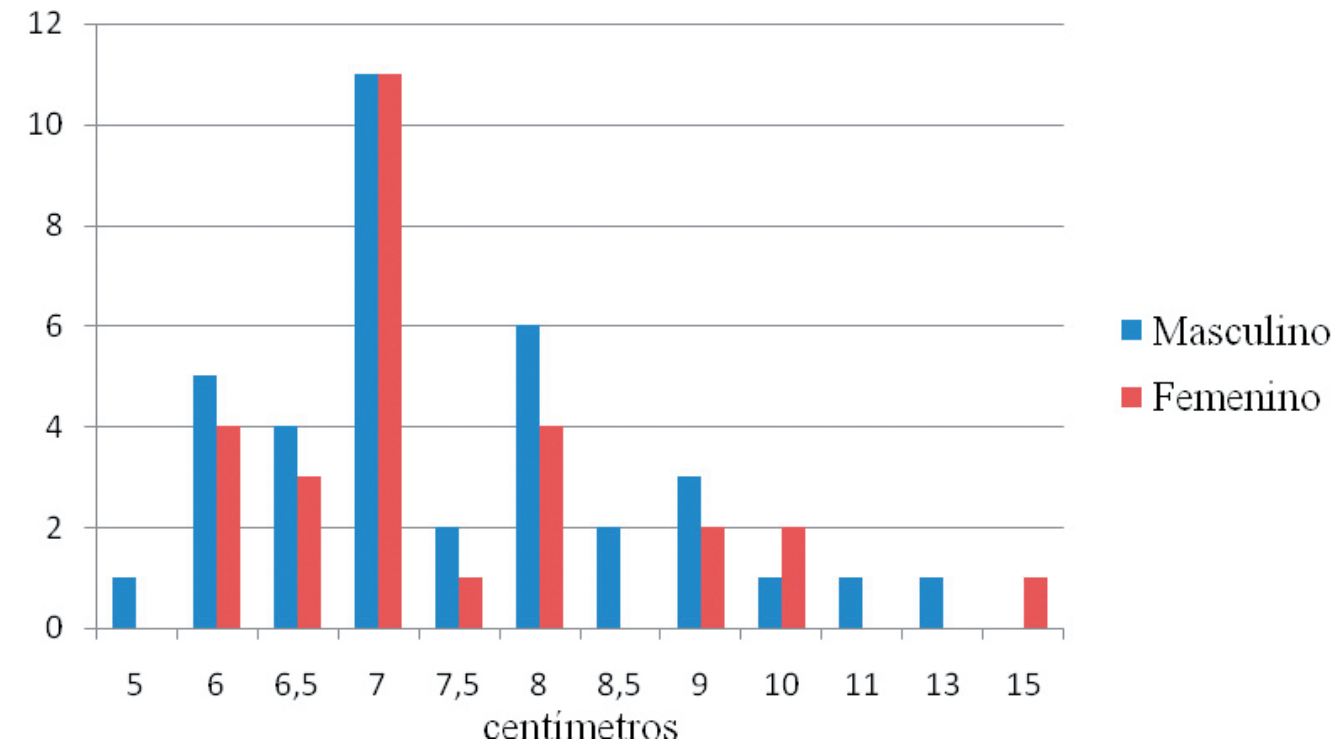

Fig. 3. Distribución de la longitud del apéndice en sexos masculino y femenino. 
Respecto del número de ramas arteriales de la arteria apendicular que alcanzó y se distribuyó por el apéndice fue de 3 $(92,3 \%)$ en 60 pacientes y de 4 en 5 de ellos $(7,7 \%)$. De los 28 pacientes de sexo femenino, $25(89,3 \%)$ tuvieron 3 arterias y de los de sexo masculino, $35(94,6 \%)$ presentaron tal característica. Los pacientes con 4 arterias fueron 2 de sexo masculino y 3 de sexo femenino.

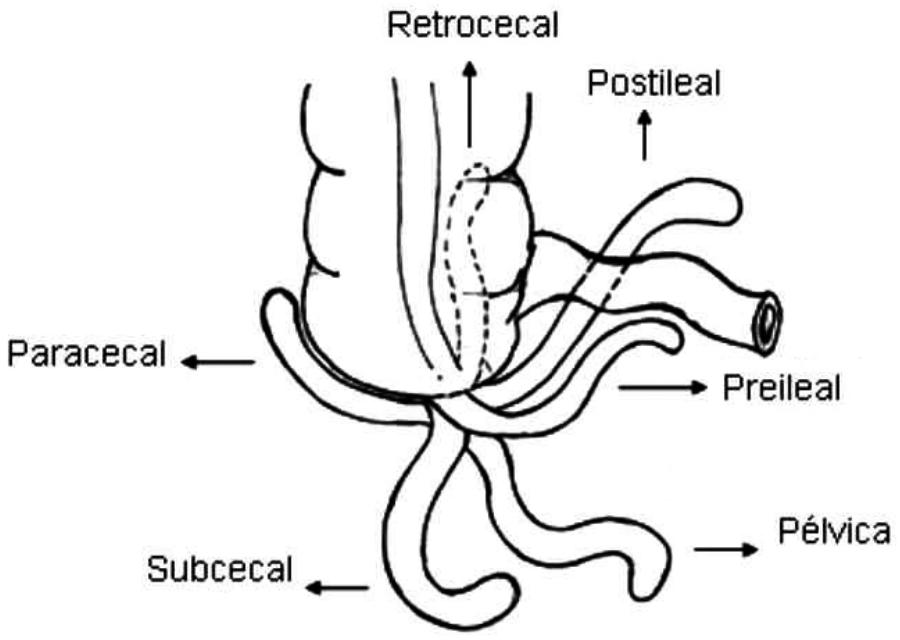

Fig. 4. Posiciones del apéndice vermiforme.

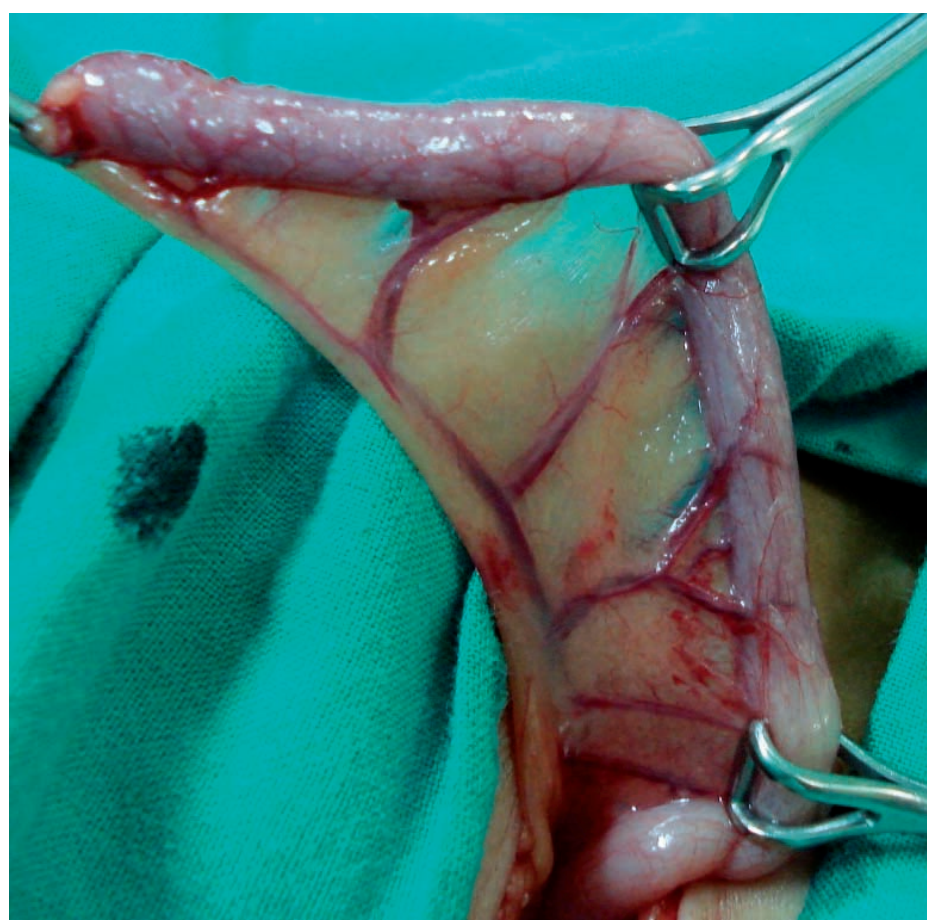

Fig. 5. Mesoapéndice y ramas de arteria apendicular.
En relación con la anatomía del mesoapéndice, éste tuvo forma triangular en $61 \mathrm{ca}-$ sos $(93,8 \%)$ y oval en $4(6,2 \%)$. El lugar de fijación del meso en el apéndice se realizó en su tercio medio en 38 casos $(58,5 \%)$ y en su tercio distal en $27(41,5 \%)$. En el sexo masculino el meso se fijó a nivel de su tercio medio en 23 casos $(62,2 \%)$ y en su tercio distal en 14 (37,8 \%); en el sexo femenino, el meso se fijó en su tercio medio en 15 casos $(53,6 \%)$ y en su tercio distal en $13(46,4 \%)$. Una vista del mesoapéndice y las ramas de la arteria apendicular se muestra en la Fig. 5.

\section{DISCUSIÓN}

Embriológicamente, el apéndice vermiforme se desarrolla a partir de un primordio llamado divertículo cecal durante la sexta semana de desarro1lo. Este esbozo del ciego es la última parte del intestino en retornar a la cavidad abdominal luego de concluir el proceso de hernia fisiológica, alrededor de la décima semana de gestación. Primero se sitúa en el cuadrante superior derecho, bajo el lóbulo derecho del hígado y posteriormente desciende a su posición definitiva en la fosa ilíaca derecha. Durante este proceso el extremo distal del esbozo del ciego da origen a un divertículo de escaso calibre, constituyendo el apéndice primitivo (Sadler, 1996).

Los primeros dibujos conocidos del apéndice se deben al gran inventor, dibujante y anatomista del siglo XVIII, Leonardo Da Vinci. La descripción del apéndice como órgano la realizó en 1521, Giacomo Berengario Da Carpi, anatomista y profesor de medicina en Bolonia. Andreas Vesalius, en su obra Acta Anatómica, publicada en 1543, también dibuja el apéndice en sus preparaciones (Pestana-Tirado \& Moreno).

En diversos estudios se han revisado las variaciones en la posición y longitud del apéndice vermiforme, en distintas poblaciones mundiales. La mayor parte de ellos se han realizado en poblaciones caucásicas. En Latinoamérica los datos son escasos y en Chile no existen publicaciones al respecto. Los estudios en su mayoría corresponden a observaciones post mortem, dentro de las cuales destaca como principal referencia el estudio realizado en Inglaterra por Wakeley (1933), quien revisó la posición del apéndice vermiforme en 10.000 autopsias. Los estudios realizados en vivo son más recientes pero aún 
son escasos y con poblaciones más acotadas. En Suecia, Grunditz et al. (1983) hicieron una revisión retrospectiva de historias clínicas; en Inglaterra, Picken et al.(1993) utilizaron Tomografía axial Computada para determinar la posición del apéndice; en USA O'Connor \& Reed (1994) y Ahmed et al. (2007) en el Reino Unido utilizaron laparoscopía para determinar la posición durante cirugías abdominales. El presente estudio, en chilenos se realizó mediante la observación directa del apéndice durante cirugía por apendicitis aguda, al igual que los estudios realizados en el medio oriente de Bakheit \& Warille (1999) y Golalipour et al. (2003).

La población chilena estudiada correspondió a sesenta y cinco niños de las regiones del Maule y Aysén (VII y XI, respectivamente), en los cuales se realizó observación directa del apéndice vermiforme previo a su extirpación durante el proceso de cirugía por sospecha de apendicitis aguda. La posición del ciego se ubicó en la fosa ilíaca derecha, siendo un porcentaje muy alto (95\%), mayor al encontrado en otras poblaciones, como por ejemplo en europeos, en los cuales Ahmed et al. observaron la ubicación del ciego en la fosa iliaca derecha en un $80,9 \%$.

Respecto de la longitud del apéndice vermiforme, en los estudios en niños chilenos fue en promedio $7,5 \mathrm{~cm}$. ESte resultado es concordante con el estudio latinoamericano realizado en Colombia por Corso et al. (2009) quienes registraron 7,1 cm y discordante con el estudio asiático de Golalipour et al., los que obtuvieron $6,3 \mathrm{~cm}$. Por su parte, los estudios realizados por Bakheit \& Warille y Ndoye et al. en individuos africanos, mostraron que los apéndices vermiformes son más largos $(9,1 \mathrm{~cm}$ y $10,6 \mathrm{~cm}$ respectivamente). $\mathrm{Al}$ intentar buscar una relación entre longitud del apéndice vermiforme y talla de los individuos incluidos en nuestra muestra, no se pudo obtener una asociación entre ambas variables. En la literatura revisada tampoco se hace referencia a algún tipo de relación al respecto, al analizar una misma muestra poblacional.

La posición más frecuente del apéndice vermiforme encontrada correspondió a la retrocecal con $47,7 \%$ seguida de la pélvica con $29,2 \%$, Ello tomando el conjunto global de la muestra tanto hombres como mujeres. Este resultado es concordante con el de Corzo et al., único estudio sudamericano, publicado en Colombia, que muestra posición retrocecal en $41 \%$ y pélvica en $28 \%$. Mantiene concordancia con los estudios en Estados Unidos de Wakeley y O'Connor \& Reed, aunque difiere en los porcentajes, ya que los de Wakeley son más altos (retrocecal, ,65\% y pélvico, $31 \%$ ) y los de O'Connor \& Reed son más bajos (retrocecal $33 \%$ y pélvica 19\%); y con el de Bakheit \& Warille (Sudán), con posición retrocecal 58\% y pélvica $21 \%$. En cambio difiere significativamente de los estudios que muestran a la posición pélvica como la más frecuente: Ahmed et al., en Inglaterra, con la posición pélvica en un $51 \%$ y la retrocecal en un 20\%; Golalipour et al. en Irán, con la posición pelvica en 33\% y la retrocecal en 32\%; Ndoye et al. en Senegal, con posición pélvica en $51 \%$ y retrocecal en $27 \%$.

Los datos obtenidos por Kumar et al. (2007) en la India, donde la posición retrocecal del apéndice fue la más frecuente $(65,3 \%)$, seguida de la posición pélvica $(31,0 \%)$, son absolutamente concordantes con lo descrito por Wakeley, porcentajes que no siendo similares a los de nuestro estudio, mantienen una relación, ya la posición retrocecal prima por sobre la pélvica.

Por su parte, O'Connor \& Reed publicaron que en los Estados Unidos la presencia de apéndice retrocecal y pélvico son muy similares. Los hallazgos en habitantes de Dakar concluyeron que las posiciones preileal y subcecal eran las más frecuentes (Ndoye et al.). Incluso hay territorios como el Reino Unido en que estudios más recientes han llevado a importantes diferencias en sus hallazgos. Es así como Picken et al. publicaron que la retroileal es la más encontrada (48 $\%$ ), en cambio Ahmed et al. publicaron una serie en la cual mediante laparoscopía la más encontrada en la población inglesa fue la pélvica. En Latinoamérica, Corzo et al. encontraron que la posición retrocecal era la más frecuente.

Al considerar el origen geográfico de los niños chilenos estudiados llama la atención la importante diferencia entre ambas regiones. En la región del Maule la posición retrocecal es claramente mucho más frecuente (55\%) versus la pélvica (18\%). En cambio en la región de Aysén la posición pélvica es levemente más frecuente que la retrocecal ( $44 \%$ versus $37 \%$, respectivamente). Esto puede estar determinado por el hecho de que la distribución por sexo de la muestra de ambas regiones es distinta. La región del Maule es mayoritariamente masculina $(67,5 \%)$, en cambio la muestra de la región de Aysén está conformada principalmente por mujeres $(57,1 \%)$. Esto tiene injerencia en el sentido de que en los varones que fueron incluidos en el estudio la posición retrocecal resultó ser más de dos veces más frecuente que la pélvica ( $54,1 \%$ versus $24,3 \%$, respectivamente). En cambio en las mujeres el resultado fue que la distribución de la posición retrocecal y pélvica fue similar $(39,3 \%$ versus $35,7 \%$ respectivamente). No fue posible encontrar otra asociación o factor que pueda explicar esta diferencia.

En relación con las características anatómicas del mesoapéndice, en su gran mayoría tuvo forma triangular (94\%). Respecto del número de ramas de la arteria apendicular, la gran mayoría ( $92 \%$ de los casos), tuvo tres ramas hacia el meso y sólo el $8 \%$ tuvo cuatro. No se encontraron referencias en relación con estas variables en la literatura revisada. 
El mesoapéndice se insertó en el tercio medio del apéndice en un $58,5 \%$ de los casos. Este resultado es similar al descrito por Golalipour et al., en Irán, en el cual un $65 \%$ de sus observaciones fueron registradas con inserción en tercio medio del apéndice; En el estudio que Rahman et al. (2009) hicieron en Bangladesh, se encontró que la inserción más frecuente también era en el tercio medio, incluso con un porcentaje de casos aún mayor (76\%). No se encontraron otras referencias a esta variable en la literatura revisada.

La vía de abordaje quirúrgico determinada en este es- tudio fue principalmente mediante laparotomía de McBurney seguida de técnica laparoscópica. La tendencia actual es utilizar cada vez en forma más frecuente esta última técnica pues permite visualizar y trabajar de mucho mejor forma la cavidad abdominal y favorece una recuperación más rápida, con una reinserción más pronta a la actividad diaria normal.

Los resultados obtenidos en esta investigación son un aporte al conocimiento de las características biométricas del apéndice vermicular en niños chilenos, considerando la escasez de trabajos anatómicos en esta área en individuos vivos.

VERDUGO, R. \& OLAVE, E. Anatomic and biometric features of the vermiform appendix in Chilean operated by acute appendicitis. Int. J. Morphol., 28(2):615-622, 2010.

SUMMARY: The biometric features of the vermiform appendix are not constant in humans, and neither is his position. Data on its variations are still limited, but show the existence of differences depending on geographic region and race of individuals. In Latin America there are no published in vivo anatomical studies as references are based only on postmortem observations. The aim of this study was to identify and describe the biometric features and anatomical positions of the vermiform appendix. For this purpose direct observations and measurements of the vermiform appendix in 65 Chilean children, of both sexes, 37 male and 28 female, operated for acute appendicitis in Maule regional and Aysen hospitals were performed. Variables such as location of the cecum, appendix position and length, shape and number of arterial branches of mesoappendix. The average age and average weight was 9.7 years and $37.5 \mathrm{~kg}$. respectively. The ceacum was localized in the right iliac fossa in 62 patients (95.4\%) and in the right flank in 3 patients. The vermiform appendix average length was $7.5 \mathrm{c}$. In the total sample the most frequent position of the vermiform appendix was retrocecal (47\%), followed by pelvic (29\%). The mesoappendix was mostly triangular, with three branch arteries and inserted mainly in the middle third of the appendix. The most common surgical approach was laparotomy McBurney, followed by laparoscopy. The laparoscopic technique has advantages related to a smaller skin incision, better exploration of the peritoneal cavity, close viewing of the organs, easier dissection and removal of the appendix with faster recovery and shorter hospital stay.

KEY WORDS: Vermiform appendix; Anatomy; Biometry.

\section{REFERENCIAS BIBLIOGRÁFICAS}

Ahmed, I.; Asgeirsson, K.; Beckinham, I. \& Lobo, D. The position of the vermiform appendix at laparoscopy. Surg. Radiol. Anat., 29:165-8, 2007.

Ashcraft, K. \& Holder, T. Cirugía Pediátrica. 2ª ed. México, McGraw-Hill Interamericana, 1995.

Ashcraft, K.; Murphy, J.; Sharp, R.; Sigalet, D. \& Snyder, Ch. Cirugía Pediatrica. $3^{\mathrm{a}}$ ed. México, McGraw-Hill Interamericana, 2002.

Bakheit, M. \& Warille, A. Anomalies of the vermiform appendix and prevalence of acute appendicitis in Khartoum. East Afr. Med. J., 76(6): 338-40, 1999.

Cárdenas, G. Etiología de la apendicitis aguda. ¿Es la posición anatómica un factor predisponente?. Rev. Col. Cirugía, 7:34-37, 1992.
Corzo, E.; Forero, P.; Amaya, L.; Bohorquez, D.; Bohorquez, S. \& Saavedra, M. Posición anatómica y longitud del apéndice vermiforme en una población de raza mestiza de la ciudad de Bucaramanga - Colombia. Rev. MedUNAB., 12(3):1-5, 2009.

Golalipour, M.; Arya, B.; Azarhoosh, R. \& Jahanshashi, M. Anatomical variations of vermiform appendix in SouthEast Caspian Sea (Gorgan-Iran). J. Anat. Soc. India, 52(2):141-3, 2003.

Grunditz, T.; Ryden, C. \& Janzon, L. Does the retrocecal position influence the course of acute appendicitis. Acta Chir. Scand., 149 (7):707-10, 1983.

Kumar, S.; Bashir, A.; Kumar, V.; Sharma, S.; Saraf, R.. Vermiform appendix and appendicitis acute. $J . K$. Science, 9(4): 2007. 
VERDUGO, R. \& OLAVE, E. Características anatómicas y biométricas del apéndice vermiforme en niños chilenos operados por apendicitis aguda. Int. J. Morphol., 28(2):615-622, 2010.

McBurney,C. The incisión made in the abdominal wall in cases of apendicitis, with a description of a new metod of operation. Ann. Surg., 20(1):38-43. 1894.

Moore, K. \& Dalley, A. Anatomía con orientación clínica. $4^{\mathrm{a}}$ ed. Buenos Aires, Panamericana, 2002.

Ndoye, J.; Ndiaye, A.; Dia, A.; Fall, B.; Diop, M. \& Sow, M. Cadaveric topography and morphometric of the vermiform appendix. Morphologie, 89(285):59-63, 2005.

Netter, F. Atlas de Anatomía Humana. 2a ed. Toronto, Masson, 1999. 540 p.

Nyhus, Ll.; Baker, R. \& Fisher, J. El dominio de la Cirugía. $3^{\mathrm{a}}$ ed. Buenos Aires, Editorial Médica Panamerica, 1999.

O'Connor, C. \& Reed, D. In vivo location of the human vermiform appendix. Clin. Anat. 7(3):139-42, 1994.

O’Neill, J.; Rowe, M.; Grosfeld, J.; Fonkalsrud, E. \& Coran, A. Pediatrics Surgery. 5 ed. St. Louis, Mosby, 1998.

Pestana-Tirado, R. \& Moreno, L. Apendicectomía transumbilical, un nuevo abordaje quirúrgico. Rev. Col. de Cirugía, 19(1):54-68, 2004.

Picken, G.; Ellis, H. \& Dixon, Ak. The normal vermiform appendix at computed tomography: visualization and anatomical location. Clin. Anat., 6(1):9-14, 1993.

Rahman, M.; Khalil, M.; Rahman, S.; Mannan, S.; Sultana, S. \& Ahmed, S. Anatomical Positions of Vermiform Appendix in Bangladeshi People. J. Bangladesh Society Physiol., 1:5 -9, 2009.

Rostion, C. Cirugía Pediátrica. Santiago, Mediterráneo, 2001.

Sabiston, D. Atlas de Cirugía. México, Interamericana McGraw-Hill, 1996.

Sadler, T.W. Lagman Embriología Médica. 7ed. México D.F.: Médica panamericana, 1996. 424 p.

Wakeley, C. The position of the vermiform appendix as ascertained by the analysis of the 10.000 cases. J. Anat., 67:277, 1933.

Williams, P.; Bannister, M.; Collins, P.; Dyson, M.; Dussek, J. \& Ferguson, M. Gray's Anatomy. 38 ed. New York, Churchill Livingstone, 1995.
Dirección para correspondencia:

Prof. Dr. Enrique Olave

Facultad de Medicina

Universidad de La Frontera

Casilla 54-D

Temuco - CHILE

Email: eolave@ufro.cl

Recibido : 23-02-2010

Aceptado:12-04-2010 